\title{
Functional But Not Structural Networks of the Human Laryngeal Motor Cortex Show Left Hemispheric Lateralization during Syllable But Not Breathing Production
}

\author{
Kristina Simonyan, ${ }^{1}$ John Ostuni, ${ }^{2}$ Christy L. Ludlow, ${ }^{1}$ and Barry Horwitz ${ }^{3}$ \\ ${ }^{1}$ Laryngeal and Speech Section, Medical Neurology Branch, ${ }^{2}$ Clinical Neurosciences Program, National Institute of Neurological Disorders and Stroke, \\ and ${ }^{3}$ Brain Imaging and Modeling Section, National Institute on Deafness and Other Communication Disorders, Bethesda, Maryland 20892
}

The laryngeal motor cortex (LMC) is indispensible for the vocal motor control of speech and song production. Patients with bilateral lesions in this region are unable to speak and sing, although their nonverbal vocalizations, such as laughter and cry, are preserved. Despite the importance of the LMC in the control of voluntary voice production in humans, the literature describing its connections remains sparse. We used diffusion tensor probabilistic tractography and functional magnetic resonance imaging-based functional connectivity analysis to identify LMC networks controlling two tasks necessary for speech production: voluntary voice as repetition of two different syllables and voluntary breathing as controlled inspiration and expiration. Peaks of activation during all tasks were found in the bilateral ventral primary motor cortex in close proximity to each other. Functional networks of the LMC during voice production but not during controlled breathing showed significant left-hemispheric lateralization $(p<0.0005)$. However, structural networks of the LMC associated with both voluntary voice production and controlled breathing had bilateral hemispheric organization. Our findings indicate the presence of a common bilateral structural network of the LMC, upon which different functional networks are built to control various voluntary laryngeal tasks. Bilateral organization of functional LMC networks during controlled breathing supports its indispensible role in all types of laryngeal behaviors. Significant left-hemispheric lateralization of functional networks during simple but highly learned voice production suggests the readiness of the LMC network for production of a complex voluntary behavior, such as human speech.

\section{Introduction}

Voice plays an important role in human communication. From its first onset at birth, the natural use of voice is for survival: vocal expressions of hunger and pain have an alarming quality, evoking a desire to assist. These genetically determined vocal reactions are controlled by the lower brainstem (Jürgens, 1995) and are present even in anencephalic infants, who lack a forebrain but have an intact brainstem (Monnier and Willi, 1953). Within the first years of life, infants begin to use voice for their first words and learn utterances for speech and song, which require the involvement of higher cortical and subcortical structures. Voluntary initiations of innate vocal patterns, such as laughter and cry, are controlled by the anterior cingulate cortex, whereas learned vocal behaviors, such as speech and song, are controlled by the laryngeal motor cortex (LMC) (Jürgens, 2002).

The human cortical laryngeal representation has been identified in the ventral part of the primary motor cortex (Foester,

Received 0ct. 1, 2009; revised 0ct. 13, 2009; accepted 0ct. 19, 2009.

This research was supported by the Intramural Research Programs of the National Institutes of Health, the National Institute of Neurological Disorders and Stroke, and the National Institute on Deafness and Other Communication Disorders. We thank Rick Reynolds and Drs. Ziad Saad and Gang Chen for their support with PPI analysis, Sandra B. Martin for her help with subjects' recruitment, and Dr. Pamela R. Kearney for medical evaluation of participants.

Correspondence should be addressed to Dr. Kristina Simonyan, Department of Neurology, Mount Sinai School of Medicine, One Gustave L. Levy Place, Box 1137, New York, NY 10029. E-mail: kristina.simonyan@mssm.edu. DOI:10.1523/JNEUROSCI.4897-09.2009

Copyright $\odot 2009$ Society for Neuroscience $\quad$ 0270-6474/09/2914912-12\$15.00/0
1936; Penfield and Rasmussen, 1950; Rödel et al., 2004; Loucks et al., 2007; Brown et al., 2008). Neuroanatomically, the laryngeal motor cortical pathway descends within the pyramidal tract, projecting bilaterally to the nucleus ambiguus of the brainstem (Kuypers, 1958), the site of laryngeal motoneurons. Due to these bilateral projections, electrical stimulation of the LMC in one hemisphere produces movements of both vocal folds (Foester, 1936) and a unilateral cortical lesion in this region rarely affects laryngeal functions (Marshall et al., 1988; Gandour et al., 1989). In contrast, bilateral destruction of the LMC renders patients unable to speak and sing, while their nonverbal vocalizations, e.g., laughter and cry, remain preserved (Foix et al., 1926). This suggests that the LMC is minimally involved in the control of innate vocalizations, but is crucial for the control of learned voluntary vocal behaviors, such as speech and song.

In addition to precise control of vocal fold movements, all types of vocalization require coordinated breathing control. Controlled prolonged expiration is necessary to maintain adequate subglottic air pressure to start and sustain voice production, while increases of subglottic pressure are necessary for voice intensity modulations. In humans, voluntary inspiration and expiration are controlled by the dorsal primary motor cortex (Foester, 1936; Colebatch et al., 1991; Ramsay et al., 1993), while expiration is additionally controlled by the ventral motor cortex (Ramsay et al., 1993), similar to the control of voice production (Loucks et al., 2007). Thus, ventral motor cortex is responsible 


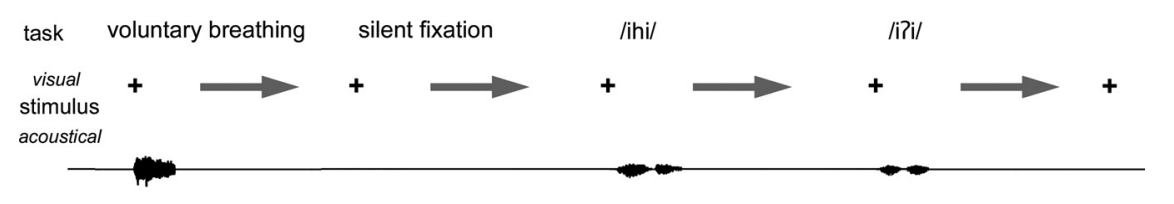

production
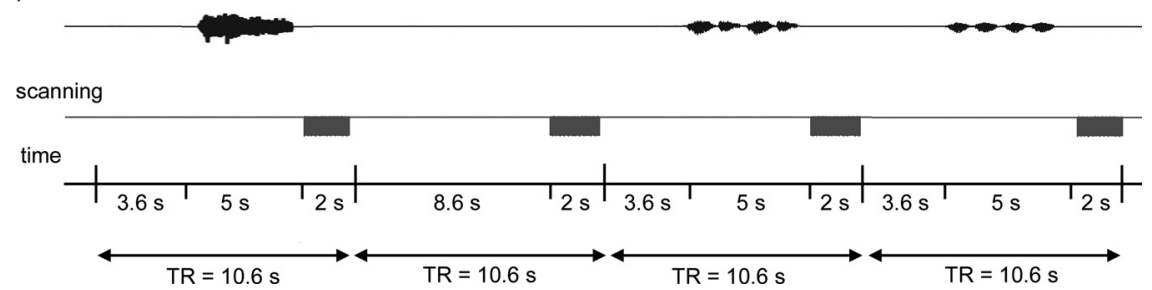

Figure 1. Schematic illustration of the experimental design in a single subject. The subject fixated at the black cross and listened to the acoustically presented sample task for a 3.6 s period. Acoustic samples were pseudorandomized and presented as syllables /i?i/, /ihi/, or single voluntary breathing. An arrow then cued the subject to produce the task within a $5 \mathrm{~s}$ period, which was followed by a 2 s period of image acquisition. No stimulus was presented for the silent fixation condition, during which the subject silently fixated at the cross and arrow.

for the integrative control of breathing during voice production for speech and song.

Despite the great importance of the LMC in the control of laryngeal behaviors, little is known about its organization in humans. We investigated the functional and structural networks of the LMC controlling two different voluntary laryngeal tasks, syllable production and controlled breathing. We hypothesized that although the structural LMC networks involved in the control of production of both syllables and breathing will be similar, significant differences will be observed in their functional networks due to the highly learned character of voluntary voice production compared with breathing.

\section{Materials and Methods}

Subjects

The same 13 right-handed (Oldfield, 1971) native monolingual Englishspeaking healthy volunteers ( 7 males/ 6 females, mean age $50.6 \pm 12.8$ years) participated in the functional magnetic resonance imaging (fMRI) and diffusion tensor imaging (DTI) studies. No participant had a history of neurological, psychiatric, voice or respiratory problems. Video fiberoptic nasolaryngoscopy was performed in all participants to confirm normal anatomy and function of the upper airways and larynx. Univariate analysis of the fMRI data from 3 subjects and measures of diffusivity [e.g., fractional anisotropy and Trace $(D)$ values] from DTI data of 6 subjects included in the present study were reported in our previous papers (Simonyan et al., 2007; Simonyan et al., 2008). Neither functional nor structural network analyses were performed or reported in these previous studies. All subjects provided written informed consent before participation in the study, which was approved by the Institutional Review Board of the National Institute of Neurological Disorders and Stroke, National Institutes of Health.

\section{Data acquisition}

Scanning was performed on a 3.0 tesla whole-body scanner (GE Excite, Milwaukee, WI) using a quadrature birdcage radio frequency head coil for acquisition of functional images and an eight-channel receive-only head coil for acquisition of diffusion-weighted images.

The fMRI study used a sparse-sampling event-related design to minimize the motion-induced artifacts during task production and to neutralize scanner noise interference with acoustic stimulus presentation. Experimental tasks included (1) two repetition of the syllable /i?i/, which consisted of the vowel /i/ as in the word "beet" followed by a glottal stop / $/$ / and then the vowel /i/ again; (2) two repetition of the syllable /ihi/, which consisted of the vowel /i/ followed by a glottal fricative / $\mathrm{h} /$ and then the vowel /i/ again; (3) controlled inspiration followed by controlled expiration, and (4) silent fixation at a cross and arrow that appeared on the screen in front of subjects' eyes. The syllable /i?i/ with a glottal stop and the syllable /ihi/ with a glottal fricative were chosen to achieve maximal vocal fold adduction and abduction, respectively. These are both used during speech production but are devoid of semantic meaning when used as syllable production tasks. The subjects were instructed to produce all tasks naturally but not to overspread their lips during production of the syllables to minimize orofacial movements during the scanning session.

For syllable and breathing production, subjects were asked first to listen attentively to the auditory sample of a corresponding task delivered through the MR-compatible headphones (Silent Scan Audio System, Avotec Inc., Stuart, FL) for a $3.6 \mathrm{~s}$ period; a visual cue (arrow) then instructed the subjects to reproduce two repetitions of the syllable /i?i/ or /ihi/ as conditions for voluntary voice production; a prolonged inspiration followed by a prolonged expiration through the mouth for controlled breathing, or silent fixation, respectively, within a $5 \mathrm{~s}$ period. No auditory stimuli were presented for the silent fixation task. Wholebrain images were acquired during $2 \mathrm{~s}$ period immediately following production of each condition (Fig. 1). Before scanning, all subjects were trained for 15 min using the experimental task design and produced all tasks accurately at the same repetition rate during the scanning sessions. Six scanning runs were acquired with a total of 36 trials per task type. All tasks were pseudo-randomized between sessions and subjects. Wholebrain functional images were acquired with a gradient-weighted echo planar imaging (EPI) pulse sequence $(\mathrm{TE}=30 \mathrm{~ms} ; \mathrm{TR}=2 \mathrm{~s}$ per volume, $10.6 \mathrm{~s}$ between volumes; $\mathrm{FA}=90$ degrees; $\mathrm{FOV}=240 \times 240 \mathrm{~mm}$; matrix $64 \times 64$ $\mathrm{mm}$; in-plane resolution $3.75 \mathrm{~mm}$; 35 sagittal slices; slice thickness $4 \mathrm{~mm}$ without gap) using blood oxygenation level-dependent (BOLD) contrast.

Whole-brain diffusion-weighted images were acquired using a single-shot spin-echo EPI sequence with 54 contiguous axial slices of $2.4 \mathrm{~mm}$ thickness, TE/TR $=73.4 / 13,000 \mathrm{~ms}, \mathrm{FOV}=240 \times 240 \mathrm{~mm}$, matrix $=256 \times 256 \mathrm{~mm}, 0.9375 \times 0.9375 \mathrm{~mm}^{2}$ in-plane resolution, and with an array spatial sensitivity encoding (ASSET) factor of 2 . Diffusion was measured along 33 noncollinear directions $(b=1000$ $\left.\mathrm{s} / \mathrm{mm}^{2}\right)$; three reference images were acquired with no diffusion gradients applied $(b=0)$.

A high-resolution T1-weighted image was collected for anatomical reference using $3 \mathrm{D}$ inversion recovery prepared spoiled gradientrecalled sequence (3D IR-Prep SPGR; TI $=450 \mathrm{~ms}$; TE $=3.0 \mathrm{~ms}$; $\mathrm{FA}=12$ degrees; bandwidth $=31.25 \mathrm{~mm}$ FOV $=240 \times 240 \mathrm{~mm}$; matrix $256 \times 256 \mathrm{~mm} ; 128$ contiguous axial slices; slice thickness 1.0 $\mathrm{mm}$; slice spacing $1.0 \mathrm{~mm}$ ).

\section{Data analysis}

Functional connectivity analysis. Functional imaging data were analyzed using AFNI software (Cox, 1996). Preprocessing included motion correction, smoothing with a $4 \mathrm{~mm}$ Gaussian filter and scaling by mean signal change at each voxel. The task-related responses were analyzed using multiple linear regression with a single regressor for each task convolved with a canonical hemodynamic response function, including the motion parameter estimates as additional regressors of no interest. The correction for multiple comparisons was made using Monte-Carlo simulations (Forman et al., 1995) that resulted in a voxelwise threshold of 0.001 and a minimum cluster size of $506 \mathrm{~mm}^{3}$ at a corrected $p \leq 0.05$. For group analysis, the anatomical images of each subject were spatially normalized to the standard Talairach-Tournoux space (Talairach and Tournoux, 1988) using the colinN27 template and the automated procedure (@auto_tlrc program), after which the resulting normalization was applied to the 4D time series datasets. To estimate the main effect of each task, group analysis was performed using a two-way within-subject mixed-effect design ANOVA with subject as the random factor and task as the fixed factor ( $p \leq 0.05$, corrected). 
Functional connectivity networks (Horwitz, 2003) were assessed using psychophysiological interaction (PPI) analysis (Friston et al., 1997; Kim and Horwitz, 2008). A PPI refers to significant changes in the contribution of one brain region's activity to that in another region due to changes in the experimental or psychological context. As the first step in PPI analysis, we identified the group peaks of activation during production of each syllable and controlled breathing in the ventral motor cortex bilaterally (i.e., laryngeal motor cortex (Rödel et al., 2004; Loucks et al., 2007; Brown et al., 2008)). The cytoarchitectonic locations of the peaks of activation during each task were identified based on the maximum probability maps (Geyer et al., 1996; Eickhoff et al., 2005); the MNI Anatomical coordinates (Eickhoff et al., 2005) of the peaks of activation are provided in the Results. These peaks of activation were used as centers of seed regions (sphere radius $4 \mathrm{~mm}$ ) to extract time series during each task production and during silent fixation in each subject. The obtained time series were further multiplied by the task vector and regressed with the time series from the entire brain in each subject. The results of regression analyses during each task production versus silent fixation indicated the changes in interaction of the seed region with the entire brain relative to the examined conditions (e.g., voice production vs silent fixation or controlled breathing vs silent fixation). A significant change in interaction meant that the functional connectivity between the seed region and a given voxel in the brain was significantly different when the subjects performed one task compared with another (e.g., voice production vs silent fixation or controlled breathing vs silent fixation). The subject-specific functional connectivity maps for each task versus silent fixation were submitted to group analyses using a two-sample $t$ test ( $p \leq 0.05$, corrected).

Using the same PPI method, post hoc PPI analyses were performed to contrast activation (1) during production of the syllable /i?i/ versus syllable /ihi/ to identify statistically significant differences in functional network organization within one type of behavior, and (2) during production of the syllable /i?i/ versus controlled breathing to identify statistically significant differences in functional connectivity between the two types of laryngeal behavior ( $p \leq 0.05$, corrected).

Probabilistic diffusion tractography analysis. Diffusion tensor modeling and probabilistic tractography were performed using the FMRIB's FDT Diffusion Toolbox implemented in the FSL software package (Behrens et al., 2007). Diffusion-weighted images were corrected for eddy current distortions and head motion using affine registration to a reference volume and an individual's T1-weighted image. The diffusion tensor for each voxel was calculated based on the eigenvectors $\left(v_{1}, v_{2}, v_{3}\right)$ and eigenvalues $\left(\lambda_{1}, \lambda_{2}, \lambda_{3}\right)$ using multivariate fitting and diagonalization. To generate a probabilistic streamline and build up the connectivity distribution, the distribution probabilities of each fiber direction were sampled in each voxel. These calculated local diffusion directions allowed for modeling of multiple fiber orientations in each voxel starting from the seed voxel. To identify the connections of the LMC with the entire brain, similar to neuroanatomical tract tracing experiments in non-human primates (Simonyan and Jürgens, 2002, 2005a), unconstrained probabilistic tractography was performed in each subject using the same peaks of activation and the same seed regions for voice and breathing production as used in the PPI analyses. The probabilistic tractography parameters were 5000 streamline samples, 2000 steps with $0.5 \mathrm{~mm}$ step length and 0.1-curvature threshold. All resulting probabilistic tractography maps were averaged to create the group maps normalized to the standard space using the colinN27 template and thresholded at a corrected $p=0.01$.

Overlap between functional and structural networks. The corrected group maps of functional and structural networks associated with voluntary voice production (syllable /i?i/) and controlled breathing were first converted into the binary maps, including both positive and negative correlations of the functional networks. Conjunction analyses were then performed to illustrate the regions of overlapping and distinct connectivity patterns between the functional and structural networks during each behavior, respectively.

Assessment of hemispheric laterality. To examine hemispheric lateralization of functional activation and functional and structural laryngeal motor cortical networks during voluntary voice production and controlled breathing, whole-hemispheric regions of interest (ROIs) were applied to each individual dataset to extract the number of significantly activated voxels in each hemisphere during task performance and the number of significantly connected voxels contributing to each functional and structural network. Statistical significance of lateralization of functional activation between the left and right hemispheres during each behavior was assessed across all subjects using paired $t$ tests at a corrected $p \leq 0.01$; significance of lateralization of functional and structural networks between the left and right seeds associated with production of syllables /i?i/ and /ihi/ and controlled breathing, respectively, was determined using paired $t$ tests at a corrected $p \leq 0.01$.

In addition to ROI analyses, we used a laterality index (LI) to examine the lateralization of functional activation during voice and breathing production as well as the lateralization of functional and structural networks associated with voluntary voice production and controlled breathing from each hemisphere. The LI of functional activation during task performance was defined as follows:

$$
\begin{aligned}
& \text { (number of activated voxels in left hemisphere } \\
& \mathrm{L} 1=\frac{- \text { number of activated voxels in right hemisphere) }}{\text { (number of activated voxels in left hemisphere }} \\
& + \text { number of activated voxels in right hemisphere) }
\end{aligned}
$$

The LI of functional and structural networks associated with voluntary voice production and controlled breathing was calculated as follows:

$$
\begin{aligned}
& \text { (number of connected voxels of } \\
& \text { left seed in left hemisphere } \\
& \text { - number of connected voxels of } \\
& \text { right seed in right hemisphere) } \\
& \mathrm{L} 1=\frac{\text { (number of connected voxels of }}{\text { (nut }} \\
& \text { left seed in left hemisphere } \\
& + \text { number of connected voxels of } \\
& \text { right seed in right hemisphere) }
\end{aligned}
$$

A positive LI was interpreted as left hemispheric lateralization and a negative LI indicated right hemispheric lateralization of activation or network (Seghier, 2008). Statistical significance of LI of functional activation was assessed using within-group one-sample $t$ test at a corrected $p \leq 0.02$; statistical significance of LI of functional and structural networks was determined using paired $t$ tests at a corrected $p \leq 0.01$.

\section{Results}

Similar to the results of other studies (Loucks et al., 2007; Simonyan et al., 2007; Olthoff et al., 2008), voluntary voice production and controlled breathing elicited typical pattern of bilateral brain activation along the Sylvian fissure, including the precentral (areas $4 \mathrm{a}, 4 \mathrm{p}$, and 6) and postcentral (areas 3a, 3b, 1 and 2) gyri, parietal operculum (OP 1-4), insula, superior temporal (STG) and supramarginal gyri, supplementary motor area (SMA), putamen, thalamus, midbrain and cerebellum. Brain activation during both tasks was largely symmetrical in both hemispheres (Fig. $2 A-C)$ based on ROI analyses $\left(t_{12}=-0.42, p=0.34\right.$ for syllable /iPi/ production, $t_{12}=1.10, p=0.29$ for syllable $/ \mathrm{ihi} /$ production, and $t_{12}=-0.79, p=0.22$ for controlled breathing) (Fig. $2 D$ ) with a mean LI of $-0.003\left(t_{12}=-0.35, p=0.74\right)$ during syllable /iri/ production, a mean LI of $0.02\left(t_{12}=0.52, p=0.61\right)$ during syllable /ihi/ production and a mean LI of $-0.02\left(t_{12}=\right.$ $-0.85, p=0.41$ ) during controlled breathing (Fig. $2 E$ ). With respect to functional localization of the LMC, the peaks of activation during both voluntary voice production and controlled breathing were found in close proximity to each other in the lower portion of the primary motor cortex. During voice production, the peaks of activation in the left and right hemispheres were localized in the area 4p (Geyer et al., 1996) (MNI Anatomical coordinates of syllable /iPi/: left $x=-50, y=$ $-14, z=40$, right $x=51, y=-15, z=41$, and syllable /ihi/: left $x=-52, y=-13, z=40$, right $x=50, y=-14, z=40)$. During controlled breathing, the peak of activation in the left 
A Production of syllable /iri/
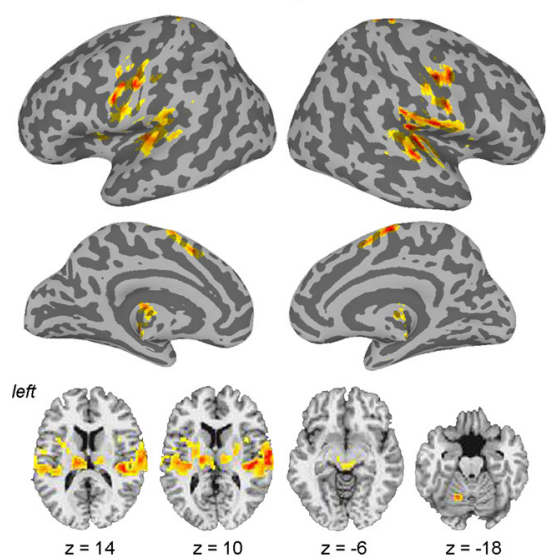

B Production of syllable /ihi/
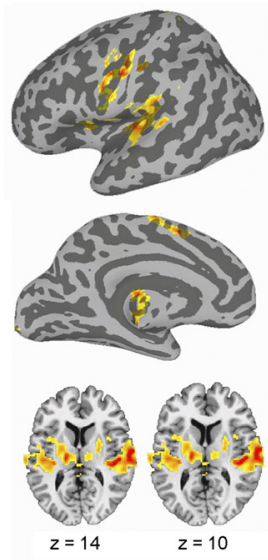
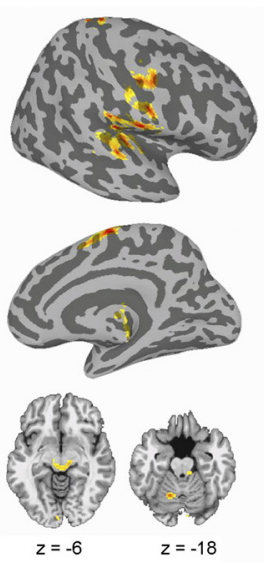

$\mathbf{E}_{\text {on: }}$

C Production of controlled breathing
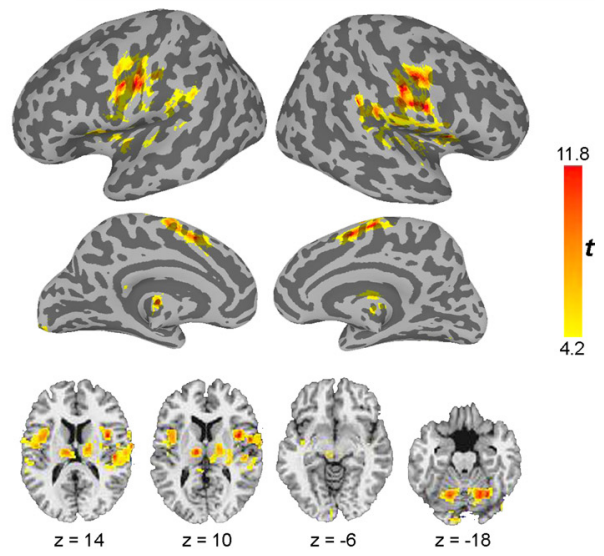
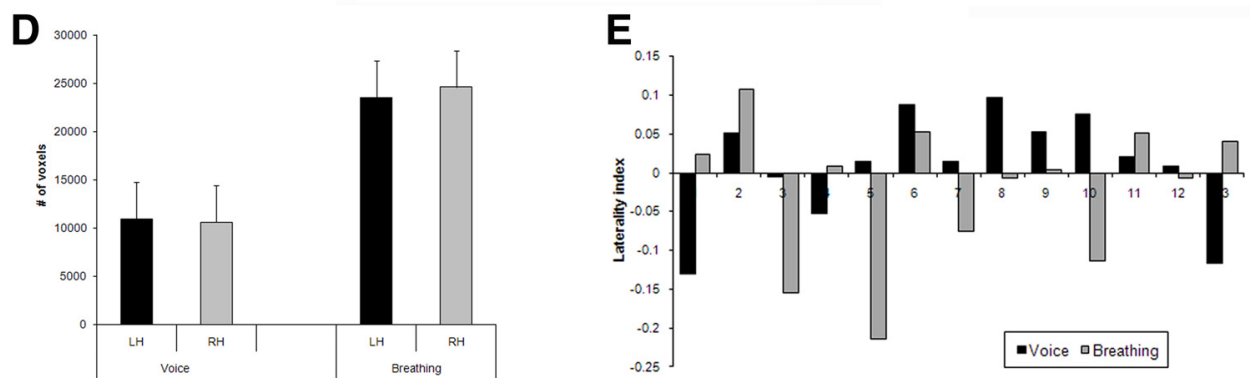

Figure 2. $\quad \boldsymbol{A}-\boldsymbol{C}$, Group maps of functional activation during production of voluntary voice production as repetition of syllables /iPi/ $(\boldsymbol{A})$ and /ihi/ $(\boldsymbol{B})$ and during voluntary breathing $(\boldsymbol{C})$. Cortical activation is presented on inflated cortical surfaces; subcortical and cerebellar activation is shown on the series of axial images of a single subject in the standard space ( $p \leq 0.05$, corrected). The color scale bar represents $t$-values (14 degrees of freedom). $\boldsymbol{D}$, Bar graphs depicting the total number of significantly activated voxels in each hemisphere during production of voice (combined syllables /iPi/ and /ihi/) and controlled breathing. Error bars represent SE. LH, Left hemisphere, RH, right hemisphere. E, Graphs depicting hemispheric Lls of functional activation during voluntary voice production (combined syllables /i?i/ and /ihi/) and controlled breathing in each subject based on the comparisons between the number of significantly activated voxels in the left and right hemisphere during each task. $\mathrm{LI}=$ (number of activated voxels in left hemisphere - number of activated voxels in right hemisphere)/(number of activated voxels in left hemisphere + number of activated voxels in right hemisphere). Values $>0$ indicate left-hemispheric lateralization; values $<0$ indicate right-hemispheric lateralization.

hemisphere was also found in the area $4 \mathrm{p}$, whereas the peak of activation in the right hemisphere was found in the area $4 \mathrm{a}$ (Geyer et al., 1996) (MNI Anatomical coordinates: left $x=$ $-52, y=-14, z=40$, right $x=58, y=-12, z=42)$.

\section{Functional networks of the laryngeal motor cortex}

Positive functional connections of the left LMC during voluntary voice production (i.e., both syllables /i?i/ and /ihi/) as indexed by PPI values were found with the bilateral ventrolateral and dorsolateral prefrontal cortex, posterior insula, parietal operculum, STG, middle temporal (MTG), angular and supramarginal gyri, the left inferior frontal gyrus (IFG) and ventral and dorsal premotor cortex on the lateral brain convexity, with the bilateral SMA proper and posterior cingulate cortex and the left pre-SMA on the medial surface, and with the bilateral putamen, globus pallidus, caudate nucleus, thalamus, midbrain, and cerebellum. Bilateral negative connections of the left LMC were observed with the ventral and dorsal primary sensorimotor cortex, anterior and middle cingulate cortex, and cerebellar vermis (Figs. $3 A, 4 A, 5 A$ ).

Functional connections of the right LMC during voluntary voice production were comparable to connections of the left LMC, but involved fewer brain regions. Compared with functional connections of the left LMC, the right LMC network did not involve the premotor cortex, IFG, operculum, pre-SMA, SMA proper, and midbrain (Figs. $3 A, 4 A, 5 C$ ).

Hemispheric differences in functional connectivity originating from the left and right LMC seed regions during vol- untary voice production were confirmed based on ROI analysis and LI calculations of the total number of positively and negatively functionally connected voxels in the ipsilateral hemisphere from the left and right seeds, respectively. Functional networks of the LMC during voluntary voice production showed significant left-hemispheric lateralization on ROI analysis (syllable /iPi/: $t_{12}=5.86, p=0.0005$; syllable /ihi/: $t_{12}=6.52, p=0.0005$ ) (Fig. $6 A, B$ ) with a mean LI of +0.2 $\left(t_{12}=6.42, p=0.0005\right)$ during syllable $/ \mathrm{i} \mathrm{i} \mathrm{i} /$ production and a mean LI of $+0.3\left(t_{12}=7.78, p=0.0005\right)$ during syllable /ihi/ production (Fig. 6G).

Positive functional connections of the left LMC during controlled breathing were observed with the left ventral and dorsal premotor cortex, IFG, ventrolateral prefrontal cortex, STG, with the right insula and with bilateral dorsolateral prefrontal cortex, SMA proper, posterior cingulate cortex, caudate nucleus, putamen, globus pallidus, thalamus, and cerebellum. Negative functional connections were found with the bilateral ventral sensorimotor cortex, anterior and middle cingulate cortex and cerebellar vermis as well as with the right dorsal primary sensorimotor cortex (Figs. 5B, $7 A$ ).

Functional connections of the right LMC during controlled breathing were similar to the left LMC network; however, the PPI values were predominantly negative. The only positive connections of the right LMC during breathing were observed with the right dorsolateral prefrontal cortex, posterior cingulate cortex, and left putamen. Bilateral negative functional connections of the right LMC were found with ventral and dorsal sensorimotor and 


\section{Production of syllable /i?i/}

\section{left seed}
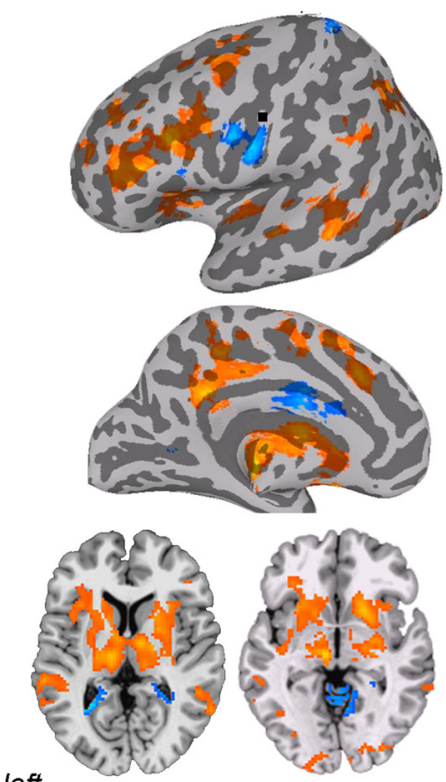

$z=7$

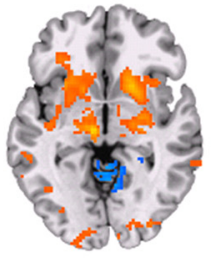

$z=-2$
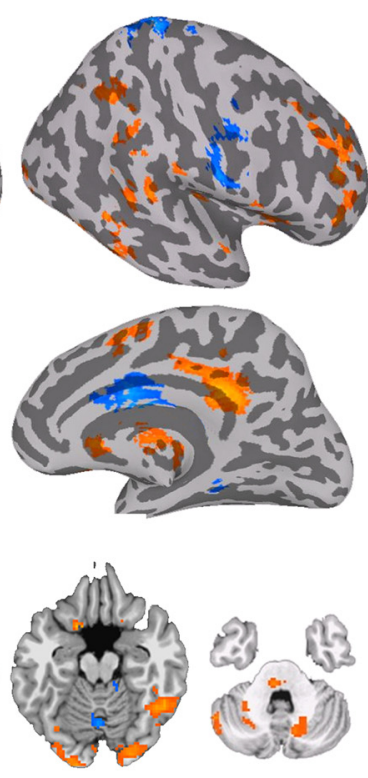

$z=-16$
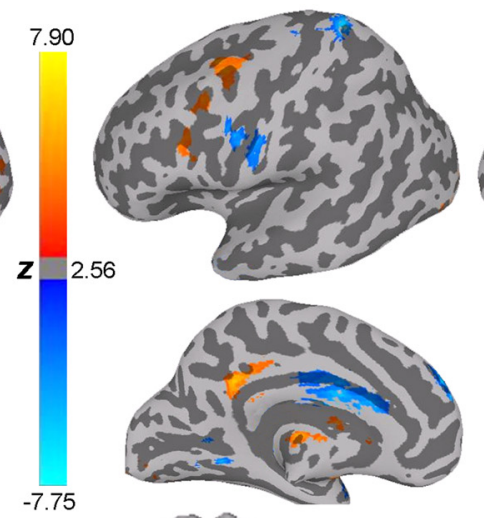

\section{right seed}
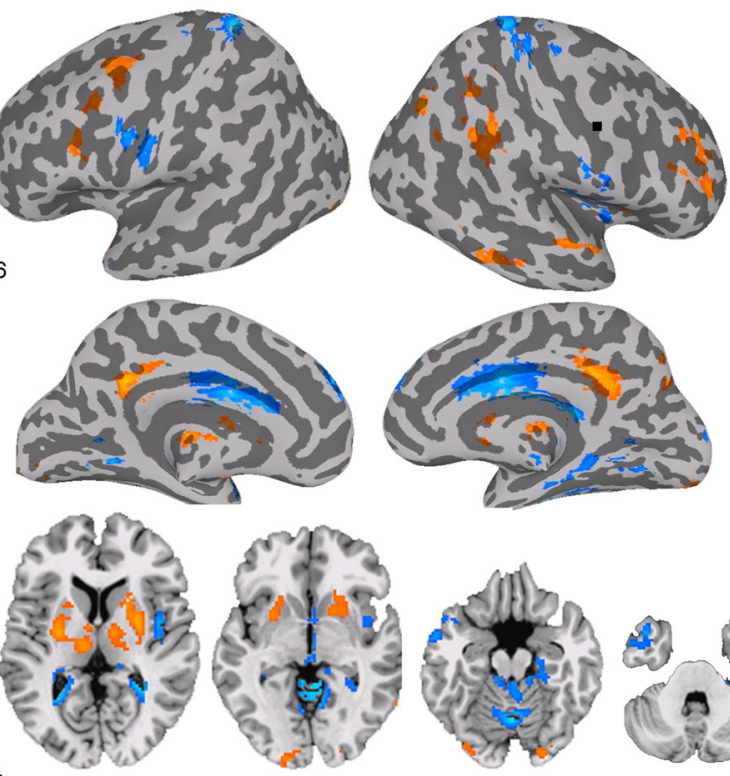

left

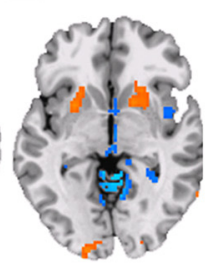

$z=-2$

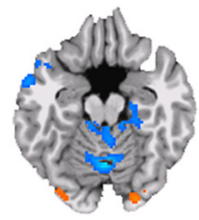

$z=-16$

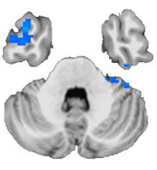

$z=-30$

\section{left seed}

\section{B Structural network}

\section{right seed}
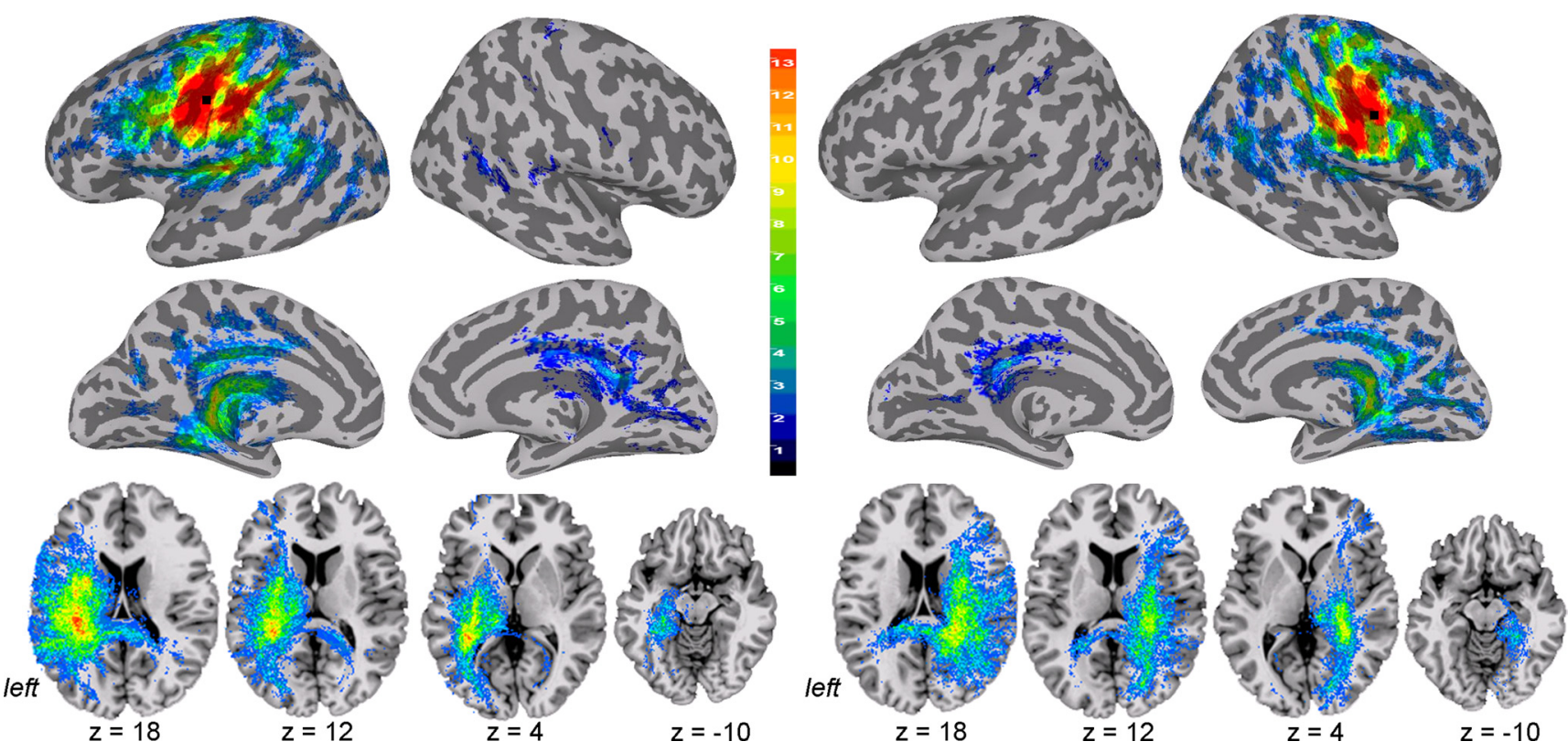

Figure 3. Functional and structural networks of the laryngeal motor cortex associated with production of syllable /i?i/. Functional connections $(\boldsymbol{A})$ and probabilistic tractography $(\boldsymbol{B})$ from both left and right hemispheric seed regions (marked as black circle) are presented on the inflated cortical surfaces; subcortical and cerebellar connections are shown on the series of axial images of a single subject in the standard space ( $p \leq 0.05$, corrected). For functional networks, color scale bar represents $z$-values, which reflect the strength of PPI connections, ranging from positive (red-yellow) to negative (light blue-dark blue). For structural networks, color scale bar illustrates the probabilistic distribution of structural connections expressed as the number of subjects (from 1 to 13 subjects) having a pathway pass through a given brain region.

premotor cortex, ventrolateral prefrontal cortex, IFG, angular gyrus, anterior and middle cingulate cortex, thalamus and cerebellar vermis (Figs. 5D, 7A). No significant functional connections of the right LMC during voluntary breathing were found with the bilateral STG, MTG, pre-SMA, and SMA proper. Func- tional LMC networks during controlled breathing appeared bilaterally distributed without statistically significant hemispheric lateralization as demonstrated by the ROI analysis $\left(t_{12}=1.78\right.$, $p=0.10)($ Fig. $6 C)$ and a mean LI of $-0.02\left(t_{12}=-1.30, p=\right.$ $0.22)$ (Fig. $6 G$ ). 


\section{Production of syllable /ihi/}

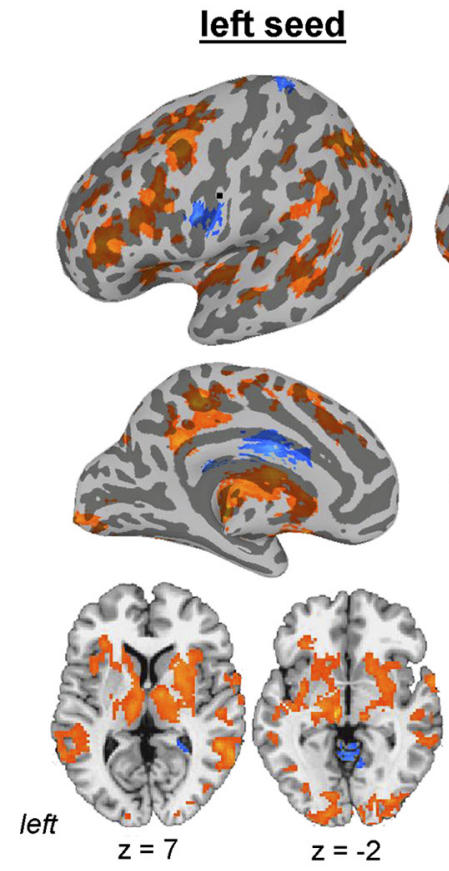

left seed
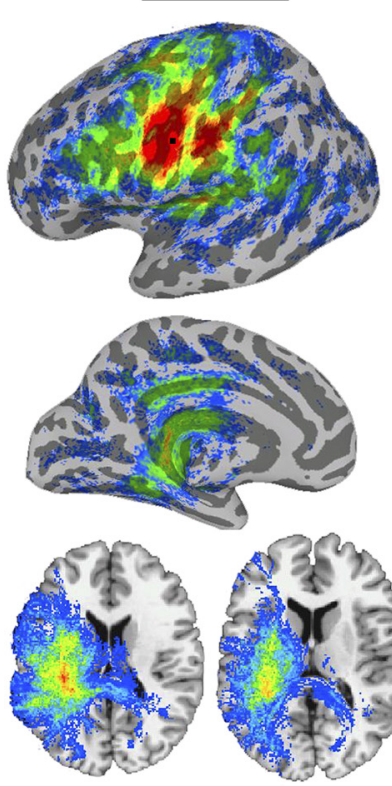

$z=18$

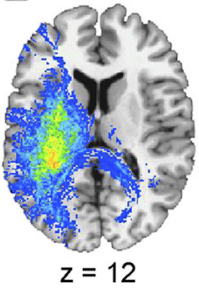

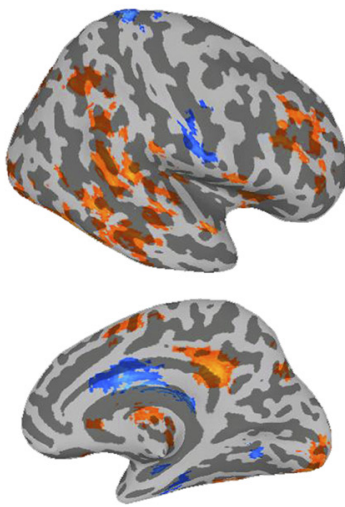
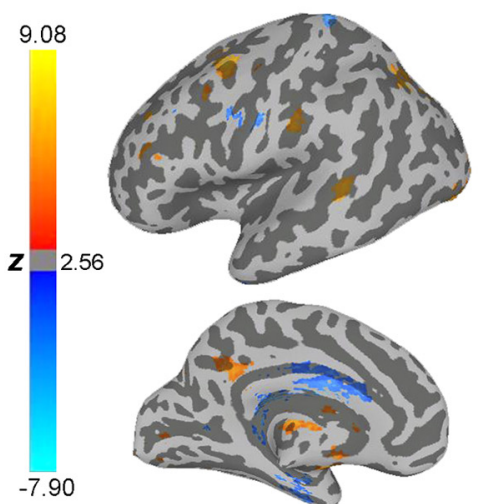

right seed
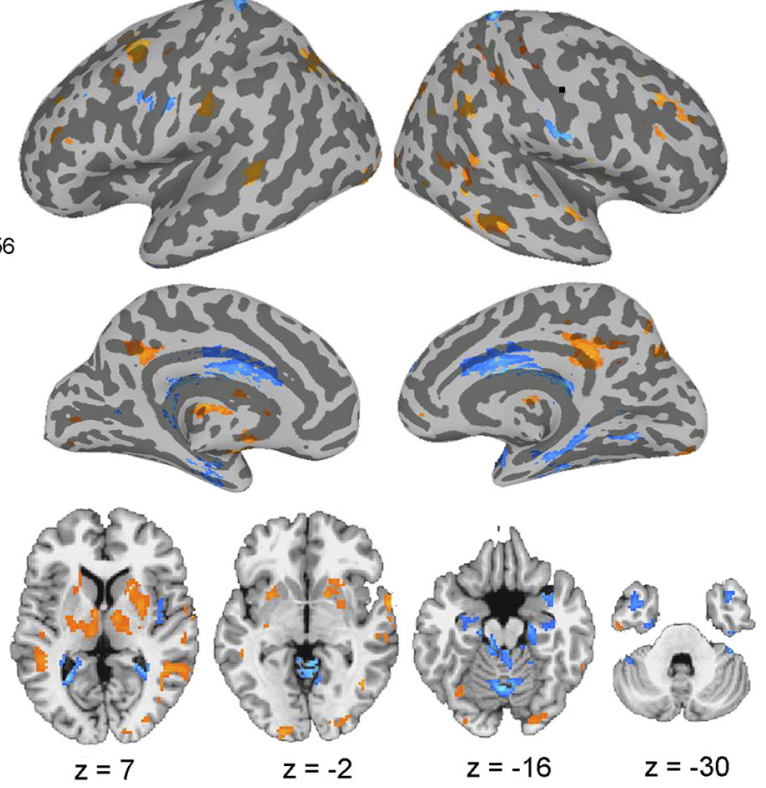

B Structural network
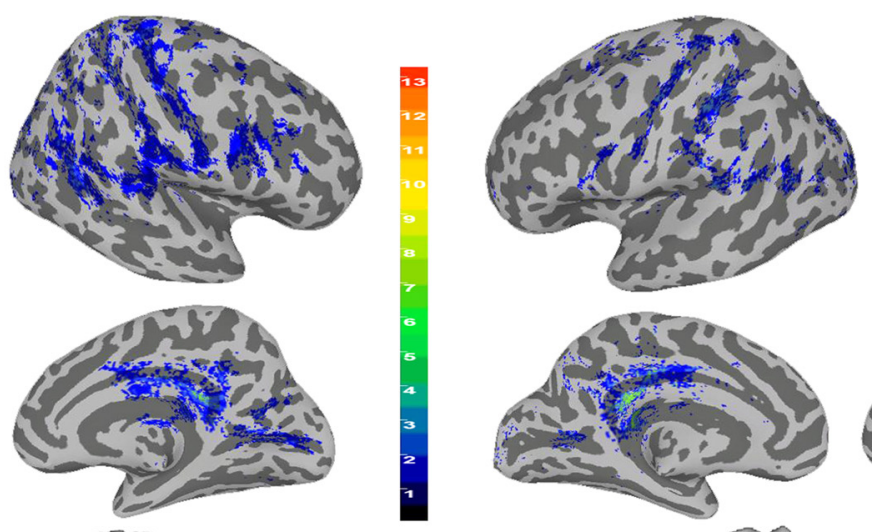

right seed
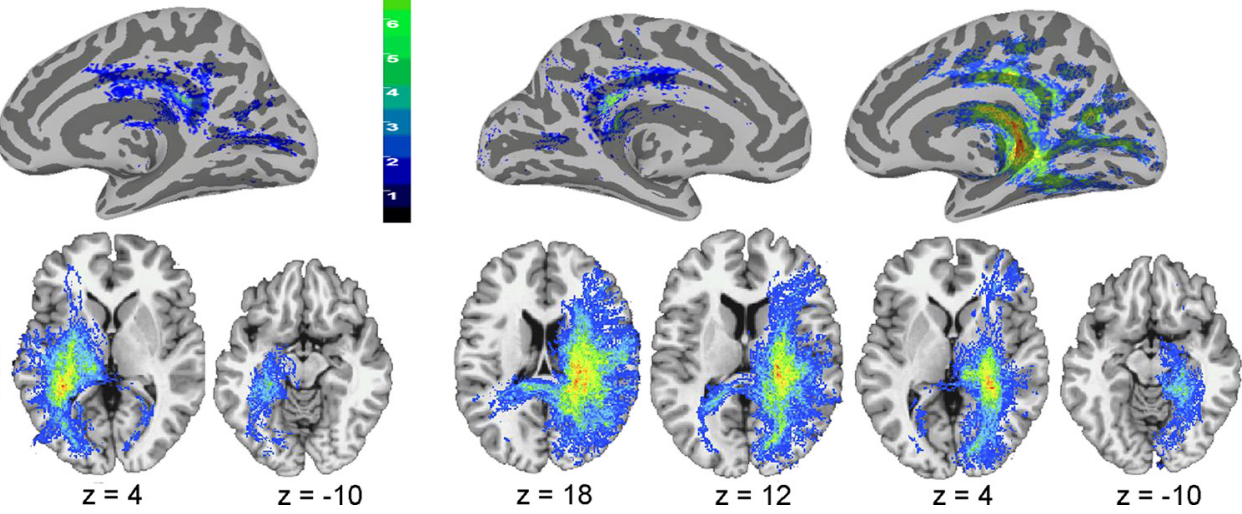

Figure 4. Functional and structural networks of the laryngeal motor cortex associated with production of syllable /ihi/. Functional connections $(\boldsymbol{A})$ and probabilistic tractography $(\boldsymbol{B})$ from both left and right hemispheric seed regions (marked as black circle) are presented on the inflated cortical surfaces; subcortical and cerebellar connections are shown on the series of axial images of a single subject in the standard space ( $p \leq 0.05$, corrected). For functional networks, color scale bar represents z-values, which reflect the strength of PPI connections, ranging from positive (red-yellow) to negative (light blue-dark blue). For structural networks, color scale bar illustrates the probabilistic distribution of structural connections expressed as the number of subjects (from 1 to 13 subjects) having a pathway pass through a given brain region.

Post hoc PPI analysis of functional networks contrasting productions of the syllable /i?i/ with the syllable /ihi/ found larger positive connections of the left LMC with the left STG and inferior parietal lobule (IPL) during production of the syllable /ihi/ (Fig. 8A).

Post hoc PPI analysis of functional networks of the left LMC during production of the syllable /i?i/ versus controlled breathing revealed significantly larger positive connections of this re- gion with the left IFG, anterior cingulate cortex, precuneus, thalamus, caudate nucleus and bilateral cerebellum during voluntary voice production (Fig. $8 B$ ).

Structural networks of the laryngeal motor cortex

Structural connections of the LMC were assessed using probabilistic diffusion tensor tractography from the same bilateral seed 
A Voice production

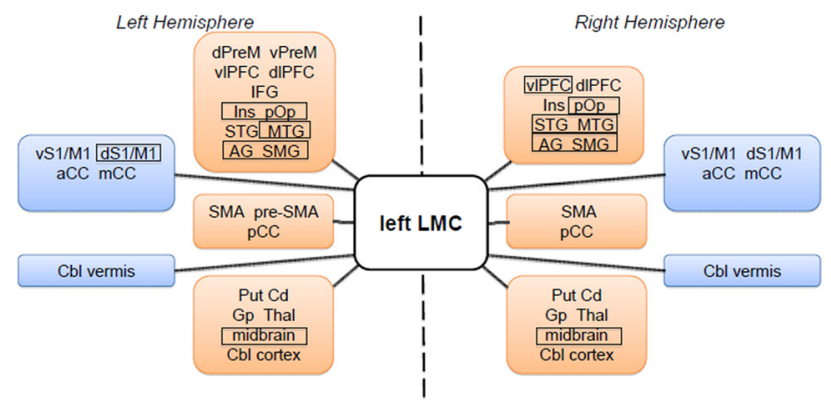

C Voice production

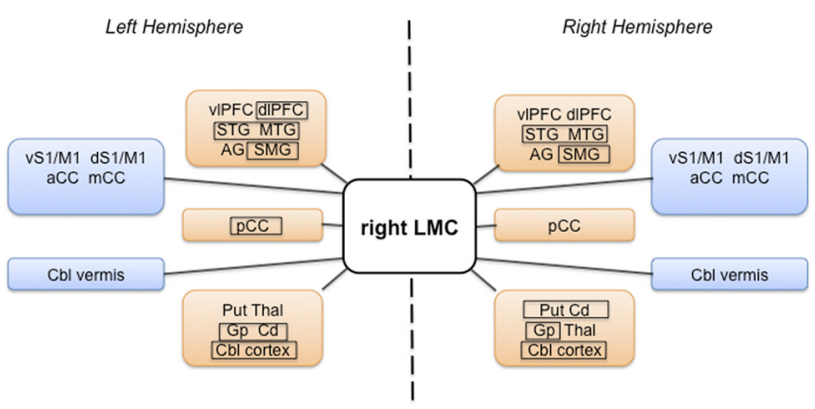

1. Functional of the laryngeal motor cortex

B Controlled breathing

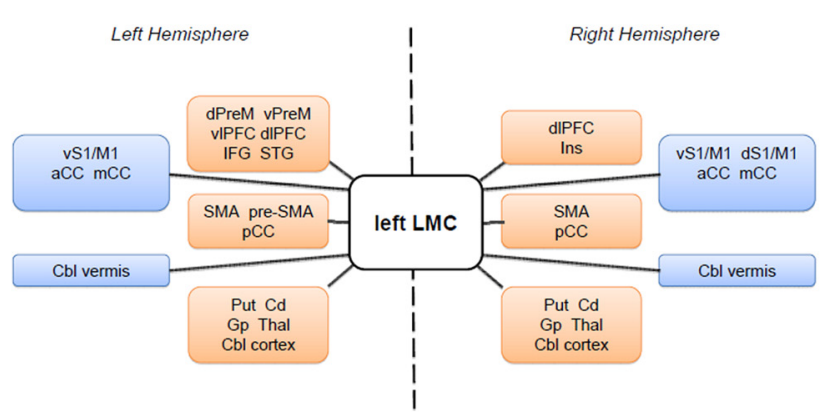

D Controlled breathing

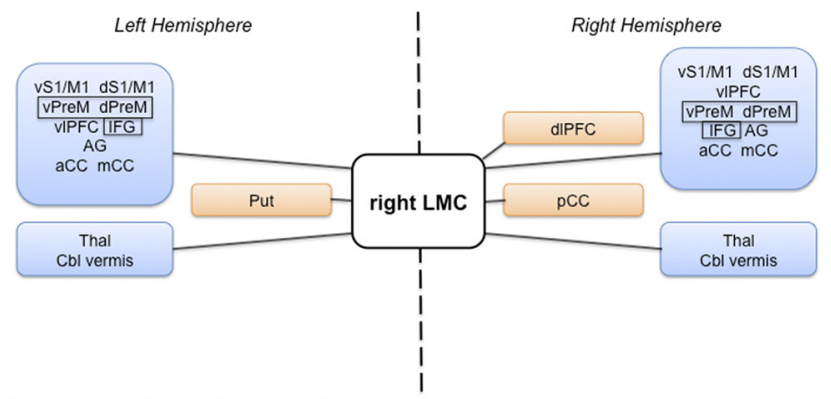

\section{Structural networks of the laryngeal motor cortex during voice production and controlled breathing}

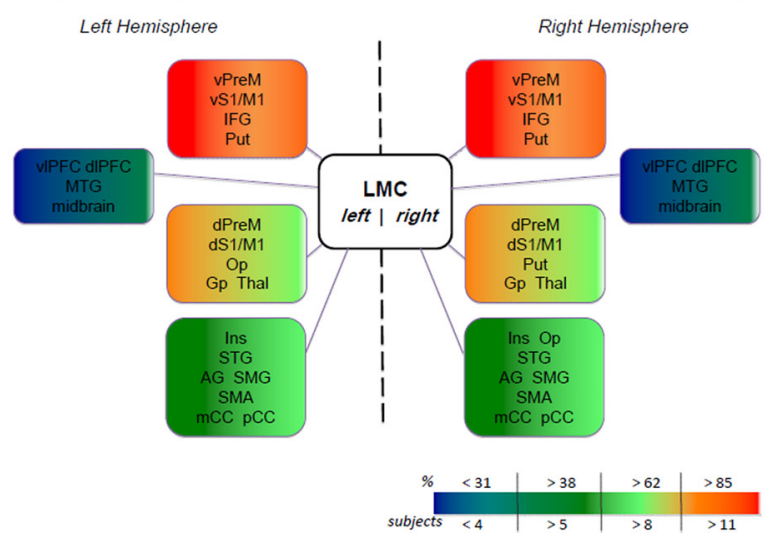

Figure 5. Block diagrams illustrating the organization of functional $(\boldsymbol{I})$ and structural $(\boldsymbol{I})$ networks originating from the left and right hemispheric seeds in the laryngeal motor cortex during production of voluntary voice (combined syllables/i?i/ and/ihi/) $(\boldsymbol{A}, \boldsymbol{C})$ and controlled breathing $(\boldsymbol{B}, \boldsymbol{D})$. $\boldsymbol{I}$, Brain regions in rectangles indicate the regions showing differences in functional networks between voice and breathing production. Regions included in the orange blocks depict positive connections of the laryngeal motor cortex, while the regions in the blue blocks indicate negative connections of the laryngeal motor cortex. II, Color-coded blocks indicate the probability of a tract passage through a given brain region. The scale represents chance of probability (percentage) of the tracts in the region and the number of subjects in whom those tracts were identified. dPreM, Dorsal premotor cortex; vPreM, ventral premotor cortex; vIPFC, ventrolateral prefrontal cortex; dIPFC, dorsolateral prefrontal cortex; Ins, insula; p0p, posterior operculum; AG, angular gyrus; SMG, supramarginal gyrus; vS1/M1, ventral primary sensorimotor cortex; dS1/M1, dorsal primary sensorimotor cortex; aCC, anterior cingulate cortex; mCC, middle cingulate cortex; pCC, posterior cingulate cortex; Put, putamen; Cd, caudate nucleus; Gp, globus pallidus; Thal, thalamus; Cbl, cerebellum.

regions associated with the peaks of activation during syllable and controlled breathing production used in the functional connectivity analyses. Structural connections of both left and right LMC associated with voluntary voice production (i.e., both syllables /i?i/ and /ihi/) showed widely distributed cortical and subcortical networks, involving unilateral ventral and dorsal premotor and primary sensorimotor cortex, IFG, ventrolateral and dorsolateral prefrontal cortex, insula, parietal operculum, STG, angular and supramarginal gyri, SMA proper, middle and posterior cingulate cortex, putamen, globus pallidus, thalamus and the midbrain (Figs. $3 B, 4 B, 5 I I$ ). Contralateral connections were observed with the primary motor and premotor cortex, operculum, posterior cingulate cortex, middle temporal and supramarginal gyri in a limited number of subjects.
Sparse structural connections of the LMC with the contralateral hemisphere in a limited number of subjects most likely represent a limitation of diffusion tensor tractography technique and, therefore, cannot be meaningfully quantified. Structural networks of the LMC associated with voluntary voice production did not show statistically significant betweenhemispheric ROI differences (syllable /i?i/: $t_{12}=0.11, p=0.92$; syllable /ihi/: $\left.t_{12}=0.02, p=0.98\right)($ Fig. $6 B, E)$ with a mean LI of $-0.01\left(t_{12}=-0.13, p=0.90\right)$ associated with production of the syllable /iPi/ and a mean LI of $-0.03\left(t_{12}=-0.36, p=\right.$ 0.73 ) associated with production of the syllable /ihi/ (Fig. $6 H$ ).

Structural connections of the LMC from the peaks of bilateral activation during controlled breathing were similar to the struc- 

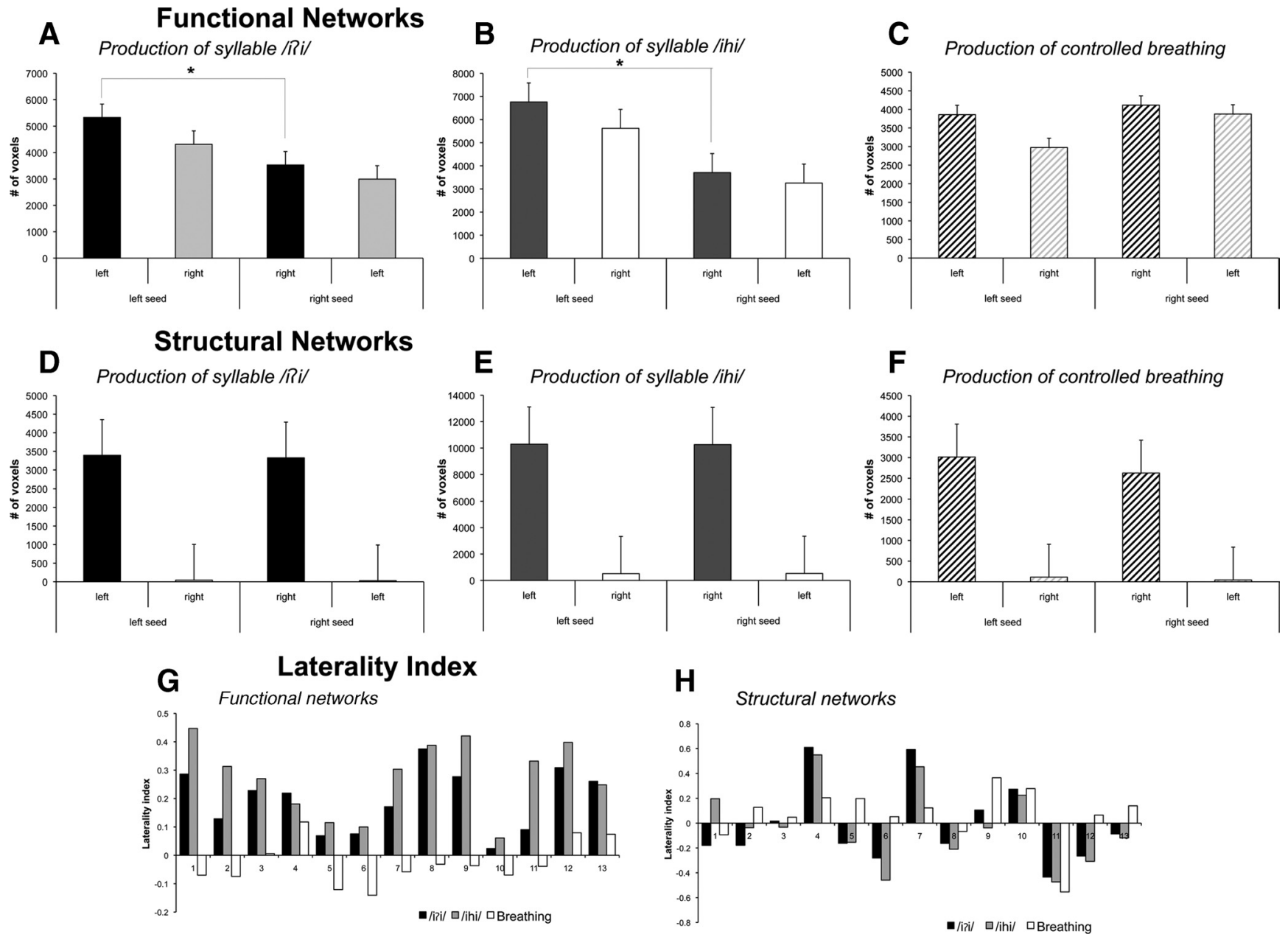

Figure 6. Bar graphs depicting the total number of voxels functionally $(\boldsymbol{A}-\boldsymbol{C})$ and structurally $(\boldsymbol{D}-\boldsymbol{F})$ connected with the left and right laryngeal motor cortex during voluntary voice production and voluntary breathing. Significant left-right seed-specific hemispheric differences (marked with an asterisk) were found for functional connections of the left laryngeal motor cortex during both types of syllable production, but not during voluntary breathing. Error bars represent SE. $\boldsymbol{G}, \boldsymbol{H}$, Graphs depicting hemispheric Lls of functional $(\boldsymbol{G})$ and structural $(\boldsymbol{H})$ networks during voluntary voice production (i.e., syllables /iii/ and /ihi/) and controlled breathing for each subject based on the comparisons between the number of voxels functionally and structurally connected with the left and right seed regions of the laryngeal motor cortex. $\mathrm{LI}=$ (number of connected voxels of left seed in left hemisphere - number of connected voxels of right seed in right hemisphere)/(number of connected voxels of left seed in left hemisphere + number of connected voxels of right seed in right hemisphere). Values $>0$ indicate left-hemispheric lateralization; values $<0$ indicate right-hemispheric lateralization.

tural networks of this region associated with voluntary voice production (Fig. 5II, 7B). Similarly, no statistically significant differences were found between the structural networks projected from the left or right LMC $\left(t_{12}=1.46, p=0.17\right)$ (Fig. $\left.6 F\right)$ with a mean LI of $+0.06\left(t_{12}=1.09, p=0.30\right)($ Fig. $6 H)$.

\section{Overlap between functional and structural networks of the laryngeal motor cortex}

When comparing functional and structural connections of the LMC, overlap between these two networks was observed in the bilateral ventral and dorsal sensorimotor cortex, angular gyrus, basal ganglia and thalamus, left ventral and dorsal premotor cortex, ventral prefrontal cortex, IFG, anterior insula, parietal operculum, middle and posterior cingulate cortex, and posterior parietal lobule during both voluntary voice and controlled breathing production (Fig. 9). However, the overlap between functional and structural networks in the right middle and posterior cingulate cortex was observed only during voluntary voice production.

\section{Discussion}

We identified structural and functional LMC networks originating from the activation peaks in the primary motor cortex during production of voluntary voice and controlled breathing in healthy humans. Our major finding is the left-hemispheric lateralization of functional networks during voice production but not breathing despite the presence of largely symmetrical bilateral hemispheric activation during both behaviors and similarly distributed motor cortical structural networks associated with these behaviors.

Although left-hemispheric lateralization of brain activation during speech production has been known since the time of Broca, bilateral hemispheric involvement has been consistently reported for less complex laryngeal behaviors, e.g., production of voice, coughing, sniffing, voluntary breathing (Ramsay et al., 1993; McKay et al., 2003; Loucks et al., 2007; Simonyan et al., 2007). We chose productions of controlled breathing and syllables with minimal linguistic meaning as simple voluntary laryngeal motor behaviors, coordination of which is necessary for speaking and singing. Both voluntary voice production and controlled breathing have similar cortical top-down central control of the laryngeal movements (Loucks et al., 2007; Simonyan et al., 2007). Nonetheless, syllable production, a highly learned task compared with breathing, appeared to be powerful enough to engage brain networks controlling more complex behavior, such as speech production. 


\section{Voluntary breathing}

left seed
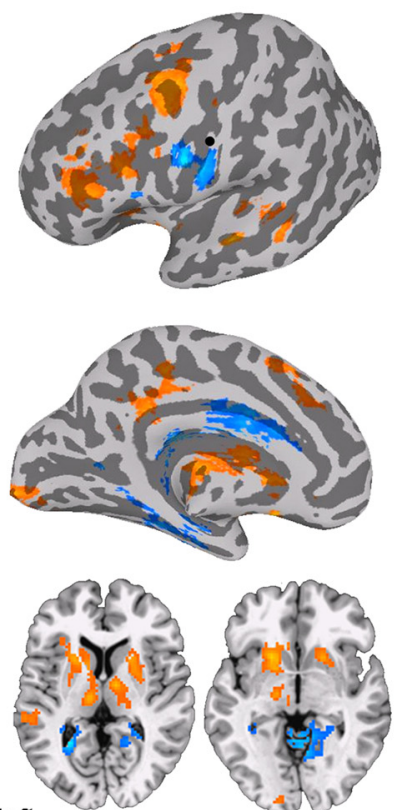

left

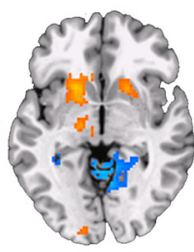

$z=-2$
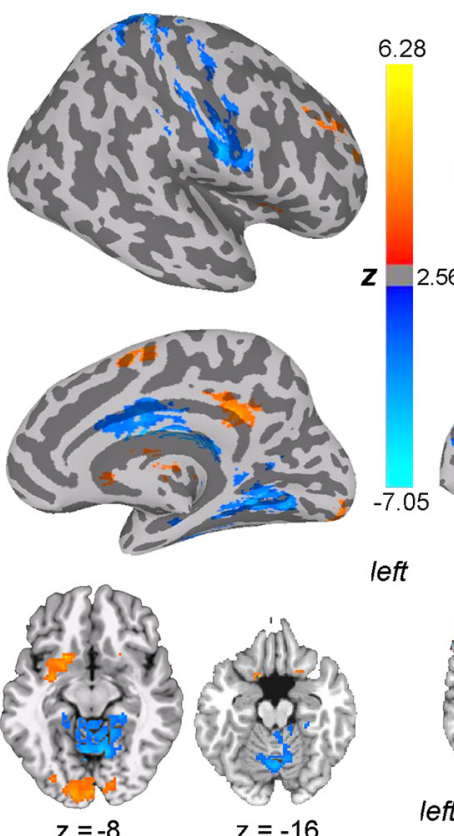

left
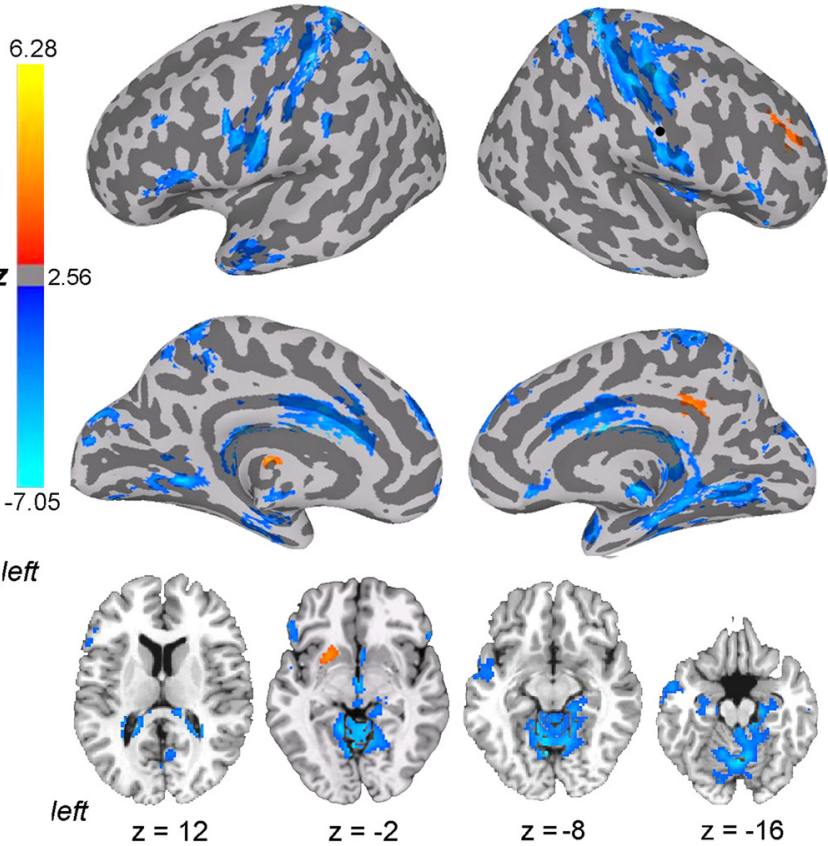

$z=-8$

$z=-16$

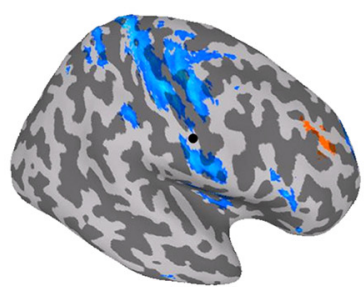

B Structural network

right seed
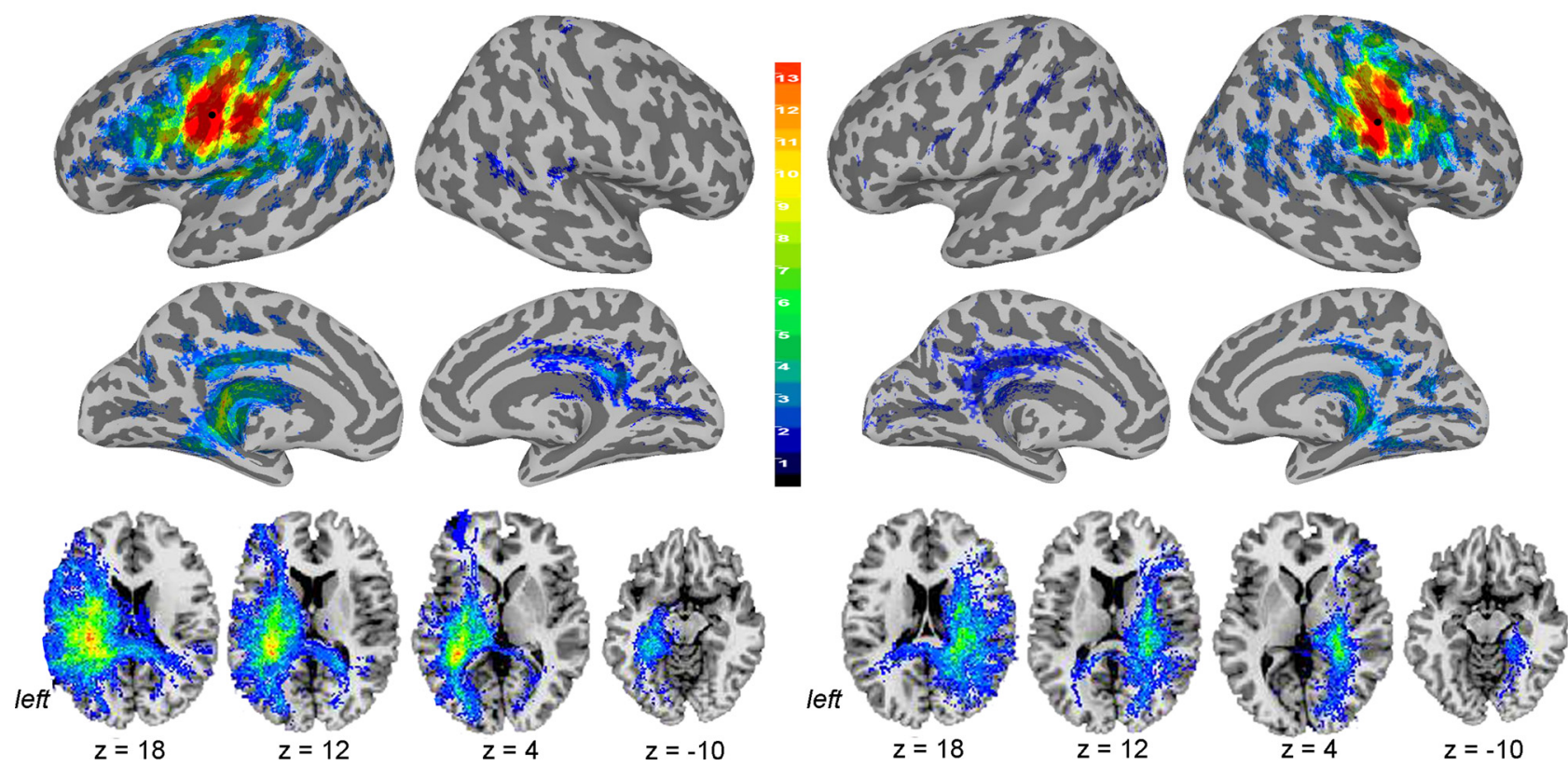

Figure 7. Functional and structural networks of the laryngeal motor cortex associated with voluntary breathing. Functional connections and probabilistic tractography from both left and right hemispheric seed regions (marked as black circle) are presented on the inflated cortical surfaces; subcortical and cerebellar connections are shown on the series of axial images of a single subject in the standard space ( $p \leq 0.05$, corrected). For functional networks, color scale bar represents $z$-values, which reflect the strength of PPI correlations, ranging from positive (red-yellow) to negative (light blue-dark blue). For structural networks, color scale bar illustrates probabilistic distribution of structural connections (e.g., the chance of probability of a pathway passing through a given brain region) ranging from 1 to 13 subjects.

Organization of functional networks for different laryngeal behaviors

Compared with bilaterally distributed LMC networks during controlled breathing, left-hemispheric lateralization of functional network during voluntary voice production was due to its predominant connections with the left IFG, STG/MTG, and SMA.
The IFG activation is most commonly associated with the production of long sequences of syllables or words (Petersen et al., 1988; Hirano et al., 1996; Horwitz et al., 2003; Ozdemir et al., 2006). No IFG activation was observed during production of either behavior in the present study; however, we found functional coupling between the LMC and IFG during both syllable 


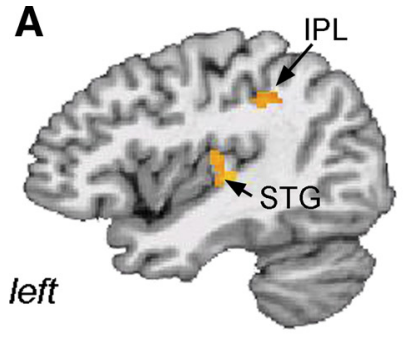

$x=39$
B

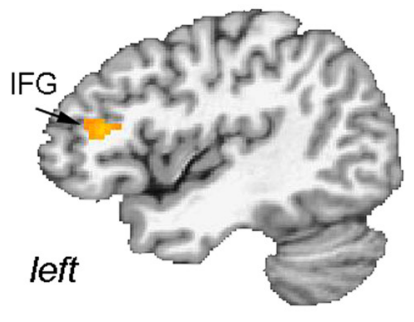

$x=41$

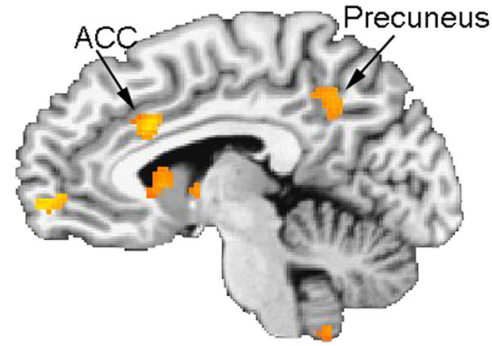

$x=6$

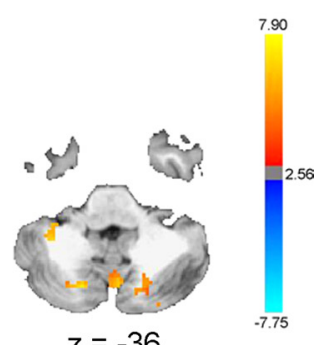

$z=-36$

Figure 8. Post hoc PPI analyses of the contrasted activation during syllable /iPi/ and syllable /ihi/ productions ( $\boldsymbol{A}$ ) and voluntary voice production (i.e., syllable /iPi/) (B) and controlled breathing productions. Significant differences in functional networks of the laryngeal motor cortex between the tasks are shown on the series of sagittal and axial images of a single subject in the standard space ( $p \leq 0.05$, corrected). Scale bars represent positive (red-yellow) and negative (light blue-dark blue) PPI values (z-score). ACC, Anterior cingulate cortex.
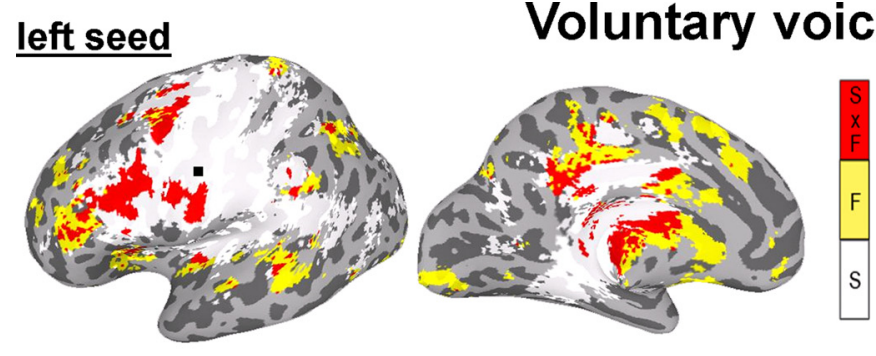

\section{production}
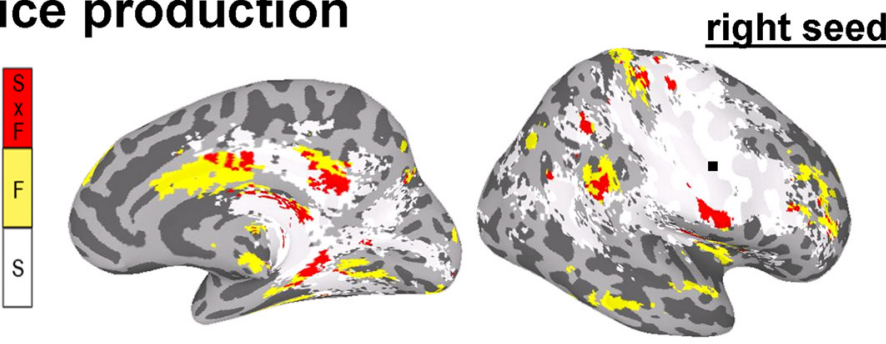

Voluntary breathing
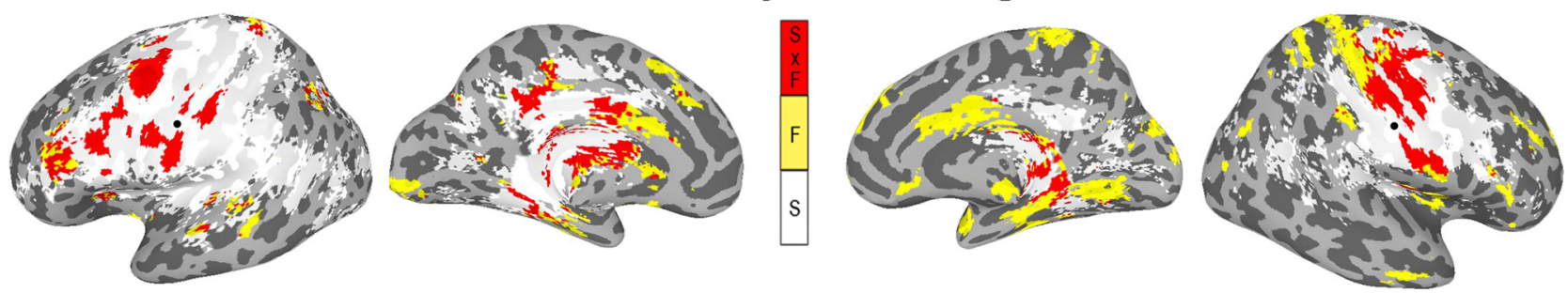

Figure 9. Group common and distinct functional and structural networks of the laryngeal motor cortex during voluntary voice production (i.e., syllable /i?i/ production) and voluntary breathing. Functional connections $(F)$ during each task are shown in yellow; structural connections $(S)$ underlying each task are shown in white; overlap between the functional and structural connections $(F \times S)$ is shown in red ( $p \leq 0.05$, corrected).

and breathing production. Our finding supports the observation of LMC-evoked activity during IFG electrical stimulation (Greenlee et al., 2004), suggesting that central processing of any component of speech production requires functional link between these two regions for speech motor preparation. However, functional connectivity with the left IFG was significantly enhanced during voice production compared with breathing, which speaks to the key role of IFG in the construction of complex functional LMC networks for control of learned (syllable production) versus innate (breathing) laryngeal behaviors.

The SMA is active during speaking, singing, simple vocalization and other voluntary laryngeal tasks (Petersen et al., 1988; Perry et al., 1999; Loucks et al., 2007; Simonyan et al., 2007; Ghosh et al., 2008). We found positive functional connections with the SMA only from the left LMC during both behaviors with a larger extent of connections with the pre-SMA during voluntary voice production. While both SMA subregions are involved in the temporal representation of motor sequences, the pre-SMA has been related to processing of higher order motor plans for subsequently ordered movement execution (Matsuzaka et al., 1992; Tanji and Shima, 1994; Shima and Tanji, 1998). This may explain the stronger functional connectivity of the LMC with
pre-SMA during learned vocalization as a component of preparation to speech production.

The STG/MTG involvement is prominent in auditory feedback control during voice production. Pitch modulations of voice feedback produce rapid adjustments in voice production to adapt to altered feedback (Houde and Jordan, 1998; Burnett and Larson, 2002; Tourville et al., 2008). Neuroimaging studies have shown that STG/MTG is more active during meaningful speech (Petersen et al., 1988; Schlosser et al., 1998; Bookheimer et al., 2000) and nonverbal vocalizations (e.g., laughter, cry) (Sander and Scheich, 2005; Sander et al., 2007) than during artificial sound perception (Binder et al., 2000). We found left-lateralized functional LMC connections during voluntary voice production with all components of the dual-stream model for speech perception (Hickok and Poeppel, 2007), suggesting that functional LMC networks controlling voluntary voice production even with minimal linguistic components have intrinsic organization for both speech perception and production.

LMC networks for voice and breathing production differed in the sign of functional connections with other brain regions. The majority of brain regions constituting functional networks of controlled breathing were negatively correlated with the LMC, 
suggesting overall less involvement of LMC networks in control of breathing. During voluntary voice production, negative functional connections of the LMC with orofacial and truncal sensorimotor cortex could be explained by the fact that the subjects produced the syllables with minimal orofacial movements and similar breathing depth, whereas negative LMC connections with the cingulate cortex may be explained by the absence of emotional expressions during syllable production. However, positive connection of the LMC with the anterior cingulate cortex when contrasting two laryngeal behaviors suggests that although simple non-emotional voice production may not require an involvement of this region, the existence of this connection may be crucial in emotional speech production.

\section{Organization of functional networks for different types of syllable production}

Functional networks during both syllables /i?i/ and /ihi/ production showed significant left-hemispheric lateralization with the only difference being larger positive connections of the left LMC with the left STG and IPL during syllable /ihi/ production.

From a motor production viewpoint, production of the glottal fricative / $\mathrm{h} /$ in the syllable /ihi/ requires additional precision of vocal fold movements for their active opening to support continued air turbulence between the vocal folds, whereas production of the glottal stop in the syllable /i?i/ only requires vocal folds hyperadduction to offset phonation. Larger connectivity of the LMC with the left STG, including Heschl's gyrus, may be associated with the preferential role of the left auditory cortex in the more complex spatiotemporal processing of rapid frequency modulations of consonant-vowel transition in the syllable /ihi/ (Price, 2000; Wise et al., 2001; Scott and Wise, 2004; Tourville et al., 2008). The greater involvement of IPL during syllable /ihi/ production may be responsible for more complex integration of sensory and motor information and temporal dynamics. As the left IPL represents a relay station between Wernicke's and Broca's areas (Catani et al., 2005), it may play a role in mapping of the acoustic-phonetic cues into lexical representations and the articulatory-gestural representation of a sample auditory stimulus for syllable production (Bohland and Guenther, 2006; Guenther et al., 2006; Jardri et al., 2007; McNamara et al., 2008). Our findings suggest that the LMC connection with STG and IPL gradually increases with the increased complexity of sound perception and production.

A limitation of the current study design should be acknowledged. While testing different laryngeal behaviors within our original experimental design limited us in time from extensive testing of different types of the same behavior (e.g., production of vowels /i/ vs /u/ vs /o/, etc.), close examination of the brain networks during different vowel production would be of a great advantage. The results of these future studies would potentially provide much needed information about subtle but important differences in the functional brain network organization controlling different components of voluntary voice production, which would ultimately lead to a much clearer insight into the organization and evolution of human speech production system.

\section{Organization of structural networks for different laryngeal behaviors}

The structural LMC connections appeared to have a similar organization with no seed-specific network lateralization during voice and breathing production. This finding supports previous data from lesion studies (Foix et al., 1926; Marshall et al., 1988; Gandour et al., 1989), which demonstrated that only bilateral
LMC lesions in humans result in elimination of voluntary learned vocal behaviors. We suggest that bilaterally descending input from the LMC to brainstem laryngeal motoneurons along with the ability of functional networks to reorganize following cortical insult may play an important role in preservation of speech during single-hemispheric LMC damage.

The structural LMC networks also showed similarity with the LMC connections in non-human primates (Jürgens, 1976; Simonyan and Jürgens, 2002, 2003, 2005a,b). The main difference between the organization of these networks was the cytoarchitectonic location of activation peaks during voice and breathing production in the primary motor cortex (area 4) (Geyer et al., 1996) in humans compared with the laryngeal muscles representation in the premotor cortex (area 6) in non-human primates (Hast and Milojevic, 1966; Hast, 1967; Hast et al., 1974). This difference in LMC location may be due to an evolutionary shift underlying the ability to control voice voluntarily for speech and song production in humans as oppose to very limited voluntary control over vocalizations in non-human primates.

\section{Summary}

Our findings suggest the presence of a common structural LMC network regardless of task production, upon which different functional networks are built to control various voluntary laryngeal behaviors. Bilateral organization of functional LMC networks during voluntary breathing supports its essential role in many motor activities, including all laryngeal tasks, e.g., speech, coughing, sniffing, and some nonlaryngeal tasks, e.g., jumping, swimming, weight lifting. Left-hemispheric lateralization of the functional networks during voluntary voice production suggests the readiness of the LMC network for a complex voluntary behavior, such as human speech. Future research should focus on characterization of the network subcomponents (e.g., specific corticocortical and corticosubcortical connections) within and between different laryngeal behaviors in healthy subjects and patients with central nervous and peripheral laryngeal damage.

\section{References}

Behrens TE, Berg HJ, Jbabdi S, Rushworth MF, Woolrich MW (2007) Probabilistic diffusion tractography with multiple fibre orientations: What can we gain? Neuroimage 34:144-155.

Binder JR, Frost JA, Hammeke TA, Bellgowan PS, Springer JA, Kaufman JN, Possing ET (2000) Human temporal lobe activation by speech and nonspeech sounds. Cereb Cortex 10:512-528.

Bohland JW, Guenther FH (2006) An fMRI investigation of syllable sequence production. Neuroimage 32:821-841.

Bookheimer SY, Zeffiro TA, Blaxton TA, Gaillard PW, Theodore WH (2000) Activation of language cortex with automatic speech tasks. Neurology 55:1151-1157.

Brown S, Ngan E, Liotti M (2008) A larynx area in the human motor cortex. Cereb Cortex 18:837-845.

Burnett TA, Larson CR (2002) Early pitch-shift response is active in both steady and dynamic voice pitch control. J Acoust Soc Am 112:1058-1063.

Catani M, Jones DK, ffytche DH (2005) Perisylvian language networks of the human brain. Ann Neurol 57:8-16.

Colebatch JG, Adams L, Murphy K, Martin AJ, Lammertsma AA, TochonDanguy HJ, Clark JC, Friston KJ, Guz A (1991) Regional cerebral blood flow during volitional breathing in man. J Physiol 443:91-103.

Cox RW (1996) AFNI: software for analysis and visualization of functional magnetic resonance neuroimages. Comput Biomed Res 29:162-173.

Eickhoff SB, Stephan KE, Mohlberg H, Grefkes C, Fink GR, Amunts K, Zilles K (2005) A new SPM toolbox for combining probabilistic cytoarchitectonic maps and functional imaging data. Neuroimage 25:1325-1335.

Foester O (1936) Motorische Felder und Bahnen. Berlin: Springer.

Foix C, Chavany JA, Marie J (1926) Diplegie facio-linguo-masticatrice d'origine cortico-souscorticale sans paralysie de membres. Rev Neurol 1:214-219. 
Forman SD, Cohen JD, Fitzgerald M, Eddy WF, Mintun MA, Noll DC (1995) Improved assessment of significant activation in functional magnetic resonance imaging (fMRI): use of a cluster-size threshold. Magn Reson Med 33:636-647.

Friston KJ, Buechel C, Fink GR, Morris J, Rolls E, Dolan RJ (1997) Psychophysiological and modulatory interactions in neuroimaging. Neuroimage 6:218-229.

Gandour J, Marshall RC, Windsor J (1989) Idiosyncratic strategies in sentence production: a case report. Brain Lang 36:614-624.

Geyer S, Ledberg A, Schleicher A, Kinomura S, Schormann T, Bürgel U, Klingberg T, Larsson J, Zilles K, Roland PE (1996) Two different areas within the primary motor cortex of man. Nature 382:805-807.

Ghosh SS, Tourville JA, Guenther FH (2008) A neuroimaging study of premotor lateralization and cerebellar involvement in the production of phonemes and syllables. J Speech Lang Hear Res 51:1183-1202.

Greenlee JD, Oya H, Kawasaki H, Volkov IO, Kaufman OP, Kovach C, Howard MA, Brugge JF (2004) A functional connection between inferior frontal gyrus and orofacial motor cortex in human. J Neurophysiol 92:1153-1164.

Guenther FH, Ghosh SS, Tourville JA (2006) Neural modeling and imaging of the cortical interactions underlying syllable production. Brain Lang 96:280-301.

Hast MH (1967) The respiratory muscle of the larynx. Ann Otol Rhinol Laryngol 76:489-497.

Hast MH, Milojevic B (1966) The response of the vocal folds to electrical stimulation of the inferior frontal cortex of the squirrel monkey. Acta Otolaryngol 61:196-204.

Hast MH, Fischer JM, Wetzel AB, Thompson VE (1974) Cortical motor representation of the laryngeal muscles in Macaca mulatta. Brain Res 73:229-240.

Hickok G, Poeppel D (2007) The cortical organization of speech processing. Nat Rev Neurosci 8:393-402.

Hirano S, Kojima H, Naito Y, Honjo I, Kamoto Y, Okazawa H, Ishizu K, Yonekura Y, Nagahama Y, Fukuyama H, Konishi J (1996) Cortical speech processing mechanisms while vocalizing visually presented languages. Neuroreport 8:363-367.

Horwitz B (2003) The elusive concept of brain connectivity. Neuroimage 19:466-470.

Horwitz B, Amunts K, Bhattacharyya R, Patkin D, Jeffries K, Zilles K, Braun AR (2003) Activation of Broca's area during the production of spoken and signed language: a combined cytoarchitectonic mapping and PET analysis. Neuropsychologia 41:1868-1876.

Houde JF, Jordan MI (1998) Sensorimotor adaptation in speech production. Science 279:1213-1216.

Jardri R, Pins D, Bubrovszky M, Despretz P, Pruvo JP, Steinling M, Thomas P (2007) Self awareness and speech processing: an fMRI study. Neuroimage 35:1645-1653.

Jürgens U (1976) Projections from the cortical larynx area in the squirrel monkey. Exp Brain Res 25:401-411.

Jürgens U (1995) Neuronal control of vocal production in non-human and human primates. In: Current topics in primate vocal communication (Zimmermann E, Newman JD, Jürgens U, eds), pp 199-206. New York: Plenum.

Jürgens U (2002) Neural pathways underlying vocal control. Neurosci Biobehav Rev 26:235-258.

Kim J, Horwitz B (2008) Investigating the neural basis for fMRI-based functional connectivity in a blocked design: application to interregional correlations and psycho-physiological interactions. Magn Reson Imaging 26:583-593.

Kuypers HG (1958) Corticobular connexions to the pons and lower brainstem in man: an anatomical study. Brain 81:364-388.

Loucks TM, Poletto CJ, Simonyan K, Reynolds CL, Ludlow CL (2007) Human brain activation during phonation and exhalation: common volitional control for two upper airway functions. Neuroimage 36:131-143.

Marshall RC, Gandour J, Windsor J (1988) Selective impairment of phonation: a case study. Brain Lang 35:313-339.

Matsuzaka Y, Aizawa H, Tanji J (1992) A motor area rostral to the supplementary motor area (presupplementary motor area) in the monkey: neuronal activity during a learned motor task. J Neurophysiol 68:653-662.

McKay LC, Evans KC, Frackowiak RS, Corfield DR (2003) Neural correlates of voluntary breathing in humans. J Appl Physiol 95:1170-1178.
McNamara A, Buccino G, Menz MM, Gläscher J, Wolbers T, Baumgärtner A, Binkofski F (2008) Neural dynamics of learning sound-action associations. PLoS One 3:e3845.

Monnier M, Willi H (1953) [The integrative activity of the nervous system of a meso-rhombencephalic anencephalus. II. Anatomical part.]. Monatsschr Psychiatr Neurol 126:259-273.

Oldfield RC (1971) The assessment and analysis of handedness: the Edinburgh inventory. Neuropsychologia 9:97-113.

Olthoff A, Baudewig J, Kruse E, Dechent P (2008) Cortical sensorimotor control in vocalization: a functional magnetic resonance imaging study. Laryngoscope 118:2091-2096.

Ozdemir E, Norton A, Schlaug G (2006) Shared and distinct neural correlates of singing and speaking. Neuroimage 33:628-635.

Penfield W, Rasmussen T (1950) The cerebral cortex of man: a clinical study of localization of function. New York: Macmillan.

Perry DW, Zatorre RJ, Petrides M, Alivisatos B, Meyer E, Evans AC (1999) Localization of cerebral activity during simple singing. Neuroreport 10:3979-3984.

Petersen SE, Fox PT, Posner MI, Mintun M, Raichle ME (1988) Positron emission tomographic studies of the cortical anatomy of single-word processing. Nature 331:585-589.

Price CJ (2000) The anatomy of language: contributions from functional neuroimaging. J Anat 197:335-359.

Ramsay SC, Adams L, Murphy K, Corfield DR, Grootoonk S, Bailey DL, Frackowiak RS, Guz A (1993) Regional cerebral blood flow during volitional expiration in man: a comparison with volitional inspiration. J Physiol 461:85-101.

Rödel RM, Olthoff A, Tergau F, Simonyan K, Kraemer D, Markus H, Kruse E (2004) Human cortical motor representation of the larynx as assessed by transcranial magnetic stimulation (TMS). Laryngoscope 114:918-922.

Sander K, Scheich H (2005) Left auditory cortex and amygdala, but right insula dominance for human laughing and crying. J Cogn Neurosci 17:1519-1531.

Sander K, Frome Y, Scheich H (2007) FMRI activations of amygdala, cingulate cortex, and auditory cortex by infant laughing and crying. Hum Brain Mapp 28:1007-1022.

Schlosser MJ, Aoyagi N, Fulbright RK, Gore JC, McCarthy G (1998) Functional MRI studies of auditory comprehension. Hum Brain Mapp 6:1-13.

Scott SK, Wise RJ (2004) The functional neuroanatomy of prelexical processing in speech perception. Cognition 92:13-45.

Seghier ML (2008) Laterality index in functional MRI: methodological issues. Magn Reson Imaging 26:594-601.

Shima K, Tanji J (1998) Both supplementary and presupplementary motor areas are crucial for the temporal organization of multiple movements. J Neurophysiol 80:3247-3260.

Simonyan K, Jürgens U (2002) Cortico-cortical projections of the motorcortical larynx area in the rhesus monkey. Brain Res 949:23-31.

Simonyan K, Jürgens U (2003) Efferent subcortical projections of the laryngeal motorcortex in the rhesus monkey. Brain Res 974:43-59.

Simonyan K, Jürgens U (2005a) Afferent cortical connections of the motor cortical larynx area in the rhesus monkey. Neuroscience 130:133-149.

Simonyan K, Jürgens U (2005b) Afferent subcortical connections into the motor cortical larynx area in the rhesus monkey. Neuroscience 130:119-131.

Simonyan K, Saad ZS, Loucks TM, Poletto CJ, Ludlow CL (2007) Functional neuroanatomy of human voluntary cough and sniff production. Neuroimage 37:401-409.

Simonyan K, Tovar-Moll F, Ostuni J, Hallett M, Kalasinsky VF, Lewin-Smith MR, Rushing EJ, Vortmeyer AO, Ludlow CL (2008) Focal white matter changes in spasmodic dysphonia: a combined diffusion tensor imaging and neuropathological study. Brain 131:447-459.

Talairach J, Tournoux P (1988) Co-planer stereotaxic atlas of the human brain. New York: Thieme.

Tanji J, Shima K (1994) Role for supplementary motor area cells in planning several movements ahead. Nature 371:413-416.

Tourville JA, Reilly KJ, Guenther FH (2008) Neural mechanisms underlying auditory feedback control of speech. Neuroimage 39:1429-1443.

Wise RJ, Scott SK, Blank SC, Mummery CJ, Murphy K, Warburton EA (2001) Separate neural subsystems within 'Wernicke's area'. Brain 124: 83-95. 